\title{
Philosophiques
}

\section{L’autonomie de la morale}

\section{Charles Larmore}

Volume 24, numéro 2, automne 1997

URI : https://id.erudit.org/iderudit/027453ar

DOI : https://doi.org/10.7202/027453ar

Aller au sommaire du numéro

Éditeur(s)

Société de philosophie du Québec

ISSN

0316-2923 (imprimé)

1492-1391 (numérique)

Découvrir la revue

Citer cet article

Larmore, C. (1997). L'autonomie de la morale. Philosophiques, 24(2), 313-328.

https://doi.org/10.7202/027453ar

\section{Résumé de l'article}

L'auteur défend la thèse selon laquelle la morale constitue un domaine de valeur irréductible. Il n'existe à l'extérieur de la morale aucun point de vue d'où la raison peut nous amener à une juste intelligence de la nature de la vie morale. Cette thèse est développée non seulement contre la perspective hobbésienne, mais aussi contre certains aspects centraux de la pensée kantienne. Au lieu d'une morale de l'autonomie, c'est en effet l'autonomie de la morale qu'il faut embrasser. Une telle conception exige pourtant que l'on abandonne une des plus grandes idéologies philosophiques de notre époque, à savoir l'image naturaliste du monde.
Ce document est protégé par la loi sur le droit d'auteur. L’utilisation des services d'Érudit (y compris la reproduction) est assujettie à sa politique d'utilisation que vous pouvez consulter en ligne.

https://apropos.erudit.org/fr/usagers/politique-dutilisation/ 


\title{
INTERVENTIONS
}

\section{L'AUIONOMIE DF LA MORALE*}

\author{
P AR \\ Charles Larmore
}

RÉSUMÉ: L'auteur défend la thèse selon laquelle la morale constitue un domaine de valeur irréductible. Il n'existe à l'extérieur de la morale aucun point de vue d'où la raison peut nous amener à une juste intelligence de la nature de la vie morale. Cette thèse est développée non seulement contre la perspective hobbésienne, mais aussi contre certains aspects centraux de la pensée kantienne. Au lieu d'une morale de l'autonomie, c'est en effet l'autonomie de la morale qu'il faut embrasser. Une telle conception exige pourtant que l'on abandonne une des plus grandes idéologies philosophiques de notre époque, à savoir l'image naturaliste du monde.

ABSTRACT: The author defends the thesis that morality constitutes an irreducible domain of value. There is no point of view external to morality from which we can reason ourselves into a proper appreciation of the nature of the moral life. This thesis is developed not only by way of objections to the Hobbesian perspective, but also in criticism of certain central aspects of Kantian ethics. Instead of a morality of autonomy, it is the autonomy of morality which we should embrace. Such a view of morality requires, however, that we break with a reigning philosophical ideology of our time, namely the naturalistic conception of the world.

Pourquoi faut-il être juste? Ce n'est pas une question que des philosophes ont simplement inventée pour eux-mêmes. Bienheureux celui qui, se sentant tiraillé entre ses obligations et ses autres intérêts, ne se demande pas parfois s'il lui faut vraiment se conformer aux exigences de la morale. Ou plutôt, moins bienheureux qu'insensible à la réalité de la condition humaine. Tout homme réfléchi, et donc chacun de nous un jour ou

\footnotetext{
NDLR Philosophiques est heureuse de publier ici le texte de la conférence d'ouverture du colloque annuel de la Société de philosophie du Québec qui s'est tenu en mai 1997 dans le cadre de l'ACFAS, à l'Université du Québec à Trois-Rivières. Ce texte important de M. Charles Larmore, invité d'honneur de la Société de philosophie du Québec, est suivi des deux commentaires qui avaient alors engagé le débat. Ceux-ci sont dus à deux spécialistes des problèmes traités par M. Larmore : il s'agit respectivement de Madame Jocelyne Couture (UQAM) et de M. Lukas S. Sosoe (Université de Montréal).
} 
l'autre, ne manquera pas de se poser cette question. Rien d'étonnant donc à ce que la question remonte à l'aube de la philosophie occidentale. Elle se trouve développée pour la première fois d'une façon systématique dans le défi que lance Glaucon à Socrate au début du Livre II de la République de Platon ${ }^{1}$ : "Montre-nous, exige-t-il, qu'il vaut toujours mieux être juste qu'injuste $n$.

Je me propose de reprendre cette question ancienne, mais non dans le dessein d'y apporter une réponse. C'est la question elle-même qui m'intéresse, ou plus exactement la façon dont la tradition philosophique l'a d'habitude interprétée. On a eu tendance, en effet, à voir dans cette question l'exigence de mettre au jour des raisons qui peuvent nous amener à embrasser la vie morale. Ainsi, on s'est appuyé sur une image fausse de la morale, car en réalité la morale constitue un domaine de valeur irréductible. C'est cette autonomie de la morale qu'il m'importe de défendre, en exposant les erreurs philosophiques qui l'ont obscurcie et en indiquant la nature d'une approche plus convenable.

En règle générale, aucune question philosophique ne permet de réponse unique. Il en est ainsi en grande partie parce que les réponses en concurrence impliquent des conceptions différentes de ce que signifie la question. C'est en effet un mème processus en philosophie que de développer une solution et que de préciser la nature du problème. Ainsi, l'objet d'évaluation ne peut être en définitive que des combinaisons de question et de réponse. Il faut juger quelle vue d'ensemble est supérieure. c'est-à-dire laquelle concorde mieux avec nos autres convictions fondamentales concernant notre situation au monde. Le cas présent n'y fait pas exception. La réponse que nous donnons à la question « Pourquoi faut-il être juste?" traduit notre conception même de la morale.

Avant de démontrer cette conclusion, je veux répéter qu'une telle question, nous nous la posons tous de temps à autre quand nous réfléchissons sur notre expérience. Par là je veux dire, non seulement qu'il s'agit d'une question universelle, mais aussi que c'est une question réflexive où l'on prend du recul pour faire de son expérience l'objet de sa pensée. Cela signifie, en particulier, que cette question surgit en général dans une situation où, ayant certaines convictions morales, nous avons découvert qu'elles se heurtent à d'autres intérêts auxquels nous ne sommes pas disposés à renoncer. Ce n'est donc pas parce que nous sommes insensibles aux considérations morales, mais parce nous sommes sensibles à d'autres choses aussi, que nous nous demandons pourquoi il nous faut être juste.

Sous ce jour-là, la façon dont bien des philosophes ont abordé cette question paraît quelque peu bizarre. On donne d'habitude à la question la forme suivante : est-ce que tout agent rationnel, connaissant les aspects essentiels de la condition humaine et ayant des besoins et intérêts normaux - mais sans aucun attachement préalable à la morale -, aurait raison de se soumettre à certaines règles d'interaction avec d'autres agents semblables? La notion de rationalité invoquée est celle qui exige qu'on choisisse les moyens les plus efficaces pour atteindre un but donné ; elle

1. Platon, République, 357b. 
implique donc que chaque agent rationnel devrait chercher à maximiser la satisfaction de ses propres besoins et intérêts. En revanche, les règles d'interaction dont il s'agit seraient de nature à défendre qu'on abuse d'autrui par la violence, des menaces ou la tromperie. Ainsi, on voit dans la question “ Pourquoi faut-il être juste? » la recherche d'un argument pour montrer que j'ai raison, sur la base même de l'efficacité avec laquelle je veux satisfaire mes propres intérêts, de limiter cette poursuite à certains égards dans mes rapports avec autrui.

Ceux qui interprètent la question de cette manière admettraient sans doute le fait sur lequel j'ai voulu insister plus haut : personne en réalité ne se pose ce problème sans avoir déjà des convictions morales. Mais ils supposent qu'on ne s'y prend bien, au niveau qu'exige du moins son traitement philosophique, qu'à condition de suspendre tout attachement préalable à la morale et de déterminer si tant est qu'il faille adopter le point de vue moral. Il est pourtant difficile, pour peu qu'on soit disposé à se distancier des constructions habituelles, d'éviter l'impression que cette façon d'interpréter la question est une erreur. N'a-t-elle pas manifestement perdu contact avec le contexte original de la question, contexte où l'on se sent déjà attaché à la morale mais attiré aussi par d'autres considérations contraires? Cette impression est juste. Et nous serons à même d'apprécier son importance, une fois mises au jour la pauvreté de cette conception de la morale et la possibilité d'une conception différente.

L'approche que je viens d'esquisser dans ses grandes lignes a reçu son premier développement systématique chez Thomas Hobbes. La formulation qu'il a donnée à la question et la sorte de solution qu'elle exige sous cette forme ont fait école depuis. Selon Hobbes, des agents rationnels ont bien raison, dans certaines conditions, de soumettre la poursuite de leurs intérêts à des contraintes qui harmonisent leurs rapports avec autrui. Le raisonnement est comme suit : dans l'absence de limites communes, les activités d'autrui constituent une menace sérieuse pour mon propre bienêtre, et évidemment il en va de même pour chacun des autres; ainsi, il est dans l'intérêt rationnel de chacun de donner son adhésion à des règles de contrainte mutuelle qui lui permettent d'atteindre ses objectifs plus efficacement qu'il ne le peut dans une situation d'anarchie.

Notoirement, Hobbes était lui-même de l'avis que ce raisonnement n'est valable que dans des circonstances spécifiques. Il fallait une autorité politique ayant la capacité de découvrir et de punir les malfaiteurs qui pourraient choisir de violer les règles pour tirer parti de la docilité de leurs voisins. Aux hobbésiens contemporains, cette condition ne paraît plus nécessaire. Si je me trouve fréquemment en contact avec d'autres personnes, si celui que je trompe aujourd'hui sera là demain pour se venger, j'aurai suffisamment raison, disent-ils, de respecter l'accord que j'ai passé avec eux. Puisqu'on a pris l'habitude de formuler l'approche hobbésienne dans le langage de la théorie des jeux et de figurer la situation initiale de menaces réciproques par le modèle du "dilemme du prisonnier ", on dit souvent que cette modification consiste à mettre en évidence le caractère "réitéré » du dilemme dans la situation en question. Cela signifie, pour l'essentiel, que chacun est supposé pris dans une série indéfinie d'interactions avec les autres individus qui l'entourent. La thèse avancée par les néo-hobbésiens est donc que dans ces circonstances, et même en l'absence d'une autorité coercitive, on a raison d'adhérer à un système de contraintes mutuelles avec ceux qui sont également prêts à y souscrire. Plus particulièrement, il serait 
rationnel de suivre dans ce cas le principe de tit for tat : on commence par coopérer et puis on répond d'une manière analogue à la réaction de l'autre (s'il coopère, on fait de même, et sinon, on use de représailles proportionnées) ${ }^{2}$. C'est sur cette base que s'est édifiée la version la plus élaborée de l'approche hobbésienne, à savoir la théorie morale de David Gauthier.

Les règles de contrainte mutuelle auxquelles on est censé arriver ont un caractère moral, cela est indéniable. Je ne veux pas contester non plus qu'il peut bien se trouver une certaine validité dans l'argument hobbésien, dont l'essentiel est que la prudence - la poursuite rationnelle de ses propres intérêts - conduit à la morale. (Bien que les philosophes se disputent à propos des détails.) Mais quelle est la conception de la morale qui est en jeu? À l'évidence, la morale se comprend ici comme un système de coopération, fondé sur l'avantage mutuel : nous avons raison, on suppose, de limiter la poursuite de nos propres intérêts parce qu'à tout prendre nous y gagnons, grâce à l'auto-limitation pareille de la part des autres.

Cette idée de la morale, est-elle pourtant satisfaisante? Pensons-nous vraiment que la morale se ramène à un système de coopération ? Pour saisir l'insuffisance de cette conception, il suffit de considérer les catégories d'individus dont elle ne tient pas compte. Ne croyons-nous pas posséder en effet des obligations morales envers ceux qui ne peuvent nous être utiles d'aucune façon, même par la réduction de leur liberté d'action? Qui plus est, ne nous reconnaissons-nous pas des obligations envers ceux que nous ne nous attendons pas à fréquenter indéfiniment, c'est-à-dire envers ceux que nous ne rencontrons qu'une seule fois sans songer à les croiser de nouveau ? La morale comme système de coopération ne peut donc expliquer un des éléments fondamentaux de notre identité morale - à savoir le respect que nous devons aux faibles et aux inconnus .

John Locke l'a bien remarqué : ‘ An Hobbist will not easily admit a great many plain duties of morality ${ }^{4}$. ("Le hobbésien sera en peine d'admettre bien des devoirs manifestes de la morale. ") Ce verdict ne devrait avoir rien d'étonnant. La réponse donnée par la conception hobbésienne à la question " Pourquoi faut-il être juste?" n'accorde de l'importance en effet qu'à ceux qui menacent notre bien-être en quelque manière et que nous pouvons envisager de rencontrer de nouveau à l'avenir. $\Lambda$ insi, compréhension de la question et réponse proposée vont de conserve. On ne se contredit pas, je veux le souligner, à voir dans la morale un système de coopération fondé sur l'avantage mutuel. Mais il faut bien se demander si c'est une conception avec laquelle on veut vivre.

2 Voir Robert Axelrod, The Evolution of Cooperation, New York, Basic Books, 1984.

3. David Gauthier, Morals by Agreement, Oxford, Oxford University Press, 1986.

4. Cité d'un manuscrit par John Dunn, The Political Thought of John Locke, Cambridge, Cambridge University Press, 1969, p. 218-219. 
C'est un trait essentiel de la conception hobbésienne qu'elle trouve à l'extérieur de la morale la base sur laquelle il faut apprécier sa valeur. Elle veut montrer comment la raison nous amène à la morale. Bien d'autres façons de répondre à la question * Pourquoi faut-il être juste? v utilisent la même tactique. Il n'est que de se rappeler toutes les théories de la vie accomplie qui prétendent justifier l'autorité de la morale, qui veulent la ramener à la visée du bonheur, de la réalisation de soi, de la perfection, ou de la tranquillité d'esprit qui est censée nous appartenir de nature.

C'est pourtant un autre chemin que je veux emprunter. Il s'agit d'une compréhension différente de la morale qui s'avère, à mon avis, plus fidèle à nos convictions les plus profondes. Je suis persuadé, en effet, que si la question "Pourquoi faut-il être juste?" se comprend de la façon que j'ai examinée jusqu'à présent, aucune réponse ne sera satisfaisante. Les croyances et les intérêts que nous avons indépendamment de toute conviction proprement morale ne suffisent pas pour nous guider jusqu'à une juste intelligence de la pensée morale. De tout point de vue situé en dehors de la morale, nous ne pouvons bien comprendre ni les exigences de la morale ni l'attitude qu'il nous faut avoir à leur égard.

La conception différente que je propose se définit par la proposition que la morale constitue un domaine de valeur irréductible. On ne peut saisir sa portée ou sa nature qu'en se situant résolument à l'intérieur du point de vue moral. Ces choses ne s'expliquent pas en termes autres que ceux qui font partie de la morale elle-même. Au lieu de tenter l'impossible et de chercher des explications extrinsèques, il nous faut donc reconnaître, c'est la thèse principale qu'il m'importe d'avancer, que la morale parle pour elle-même, pour peu que nous voulions l'écouter. L'endroit où elle parle, c'est notre conscience. Et quant à l'autorité de la conscience, elle ne découle pas de considérations indépendantes de la morale, qui seraient à même de l'authentifier. Au contraire, son autorité vient d'elle-même.

Telle est la conception de la morale qu'expose le Vicaire savoyard dans la profession de foi éloquente que Jean-Jacques Rousseau lui prête dans son Émile. Le Vicaire en énonce l'essentiel dans l'axiome selon lequel « la conscience dépose pour elle-même ${ }^{5}$. Cette réorientation de la pensée morale a exercé à son tour une influence profonde sur Emmanuel Kant. Bien des années avant la parution de ses trois Critiques célèbres, Kant a remarqué qu'à cet égard "Rousseau hat mich zurecht gebracht " ". " Rousseau m'a remis sur la bonne route. m) Malgré tous les rouages conceptuels et l'argumentation tortueuse qui composeront son éthique, Kant n'a voulu jamais abandonner l'idée que le rôle de la philosophie ne consiste pas à montrer que nous avons des raisons extrinsèques d'être juste. Notre fidélité à la morale ne peut provenir que de la conscience morale elle-même. Qu'il ait réussi à rester fidèle à ce principe, c'est là, nous le verrons, une autre question.

Pour embrasser cette conception de la morale, on n'a pas besoin, me semble-t-il, de supposer que la conscience constitue une faculté innée. On peut admettre, ce qui n'est d'ailleurs que raisonnable (quoique Rousseau et

5. Jean-Jacques Rousseau, CEuvres complètes, Paris, Gallimard, 1969, vol. 4, p. 600.

6. Cité d'un manuscrit par Ernst Cassirer, Rousseau, Kant, and Gothe, Princeton, Princeton University Press, 1945, p. 1. 
Kant eux-mêmes le passent plutôt sous silence), que notre sensibilité morale prend forme au moyen du processus de socialisation. Ainsi, elle reçoit sans doute une coloration qui reflète des préoccupations particulières de notre propre culture. Mais l'essentiel, c'est de reconnaître que la conscience seule et de son propre chef nous rend intelligible la nature des valeurs morales.

Quel sera le visage de la philosophie morale, si l'on accepte de suivre le chemin que je viens d'ébaucher? On peut ètre tenté de croire que, une fois abandonné le projet séculaire qui veut montrer comment la raison nous amène à la morale, il ne lui reste plus rien à faire. C'est cette impression qui, au début du siècle, a poussé le philosophe anglais $H$. A. Prichard, par suite de réflexions semblables, à se demander, dans le titre même d'un essai désormais célèbre, "la philosophie morale repose-t-elle sur une erreur? ?"

Toute conclusion de la sorte est pourtant précipitée. Au minimum, il y aura (Prichard lui-mème l'admet) une importante tâche négative à laquelle on ne pourra renoncer. Il faudra faire face à toutes les conceptions qui, d'une façon ou d'une autre, ne veulent pas voir dans la morale un domaine de valeur irréductible. De telles conceptions - qu'elles se mettent à nier directement la voix de la conscience ou qu'elles cherchent à expliquer la pensée morale en termes d'autres intérêts - sont en effet toujours présentes. Certaines ne relèvent que de la mode, d'autres représentent des tentations permanentes, mais il est de toute façon incontestable qu'elles peuvent exercer une séduction sur notre pensée. Au philosophe moral revient donc en premier lieu une mission de vigilance. Il est sommé de mettre au jour les différentes formes d'erreur qui peuvent nous empêcher de comprendre la morale telle qu'elle est en elle-même.

La philosophie morale a d'autres tâches aussi, tâches plus positives dont certaines nous occuperont plus tard. Mais à présent, c'est son rôle négatif, défensif peut-on dire, que je veux poursuivre. Et cela précisément à propos de Kant, dont j'ai invoqué la sagacité précédemment. Je tiens à démontrer que c'est dans le principe de l'autorité intrinsèque de la conscience et non dans l'idéal de l'autonomie, lequel en est indépendant, que se trouve la part de vérité dans l'éthique kantienne. $\grave{A}$ mon avis, l'autonomie ne fait partie intégrante de la morale sous aucun des deux rapports où dans le sillage de Kant on veut souvent la voir. Ce n'est pas en termes de l'auto-législation de la raison qu'il faut expliquer l'objectivité des jugements moraux. Et on ne peut faire du souci d'autonomie notre raison ou motif d'être juste sans retomber précisément dans la mème sorte d'erreur - la subordination de la morale à des considérations extrinsèques - dont Kant lui-même s'est proclamé guéri par les propos du Vicaire savoyard. C'est par la démonstration de cette deuxième thèse que je commence.

7. L'essai (1912) a été repris dans H. A. Prichard, Moral Obligation, Oxford, Oxford University Press, 1968, p. 1-17. 
D'abord, une remarque d'ordre méthodologique. En général, ceux qui ne veulent voir de sens dans la morale qu'à condition que ce soit la raison qui nous y amène sont obligés de proposer, comme point de départ, une notion plausible de l'homme rationnel. Cela implique qu'il faut, non seulement définir l'idée de rationalité en jeu, mais aussi présenter une image des mobiles fondamentaux de notre humanité, image à laquelle on pourrait aisément s'identifier. Ces mobiles sont censés fournir les matériaux que la raison doit conduire à la morale. Si l'on ne peut s'y reconnaître, l'argument pour la morale ne peut donc réussir.

Dans cette optique, l'attrait permanent de la conception hobbésienne s'explique facilement. Chacun se sent touché par l'image de l'homme qui veut maximiser la satisfaction de ses besoins et intérêts donnés. Or, il en va de même pour une autre façon de montrer que la raison nous amène à la morale, à savoir celle qui se réclame de l'héritage kantien. Cette conception veut faire remonter l'autorité de la morale à une idée de l'homme comme être libre. De là, la séduction qu'elle continue d'exercer sur les esprits, grâce au prestige immense dont jouit l'idéal de liberté dans notre culture.

Bien entendu, la notion de liberté peut signifier des choses différentes. Lorsque, dans la tradition kantienne, on suppose qu'elle peut servir de fondement de la morale, on y voit d'ordinaire une capacité à deux aspects. Elle comprend en premier lieu (moment négatif) le pouvoir, exercé en un mouvement de réflexion, de prendre du recul par rapport à tout ce que notre culture et histoire, tout ce que notre expérience a fait de nous ; de sorte qu'ensuite (moment positif) nous puissions nous comporter et vivre selon des règles que nous nous imposons à nous-mêmes. Dans ce sens, liberté signifie " auto-détermination " ou " autonomie ". L'idée essentielle n'est pas simplement que c'est à nous de décider si nous allons nous comporter selon des principes moraux. C'est là une banalité. Il s'agit plutôt de la notion (qui ne va pas du tout de soi) que ces principes ne sont valables que dans la mesure où ils découlent de notre capacité à vivre selon des règles que nous nous prescrivons à nous-mêmes. Ou plus exactement, leur autorité est censée résulter de la pratique rationnelle de cette sorte de liberté.

Selon cette conception kantienne, comme selon la conception hobbésienne, c'est donc la raison qui nous amène à la morale. On comprend autrement, il est vrai, les termes de la démonstration. On assimile notre humanité, non plus à la simple poursuite de nos intérêts et besoins donnés, mais au souci spécifique qu'est l'exercice de l'autonomie. Et on voit dans la raison la volonté, non de maximiser la satisfaction de préférences, mais de s'imposer des principes qui s'accordent avec, voire affirment, cette capacité de régler soi-même sa conduite. Toujours est-il que la structure de l'argument est la même : c'est à partir d'une position située à l'extérieur de la morale qu'on veut fonder son autorité. La conception kantienne se voit développée, à des registres différents, par de nombreux philosophes contemporains. Je pense par exemple à Alan Gewirth et Christine Korsgaard aux États-Unis, et à Luc Ferry et Alain Renaut en France ${ }^{8}$.

8. Alan Gewirth, Reason and Morality, Chicago, University of Chicago Press, 1978 ; Christine Korsgaard, The Sources of Normativity; Cambridge, Cambridge University Press, 1996 ; Luc Ferry, L'homme-Dieu ou le sens de la vie, Paris, Grasset, 1996 ; Alain Renaut, Qu'est-ce que le droit ?, Paris, Vrin, 1992. 
L'image de l'homme autonome joue ici, il est clair, le rôle d'un idéal. L'autonomie n'est en effet pas automatique. Au contraire, il faut apprendre à écarter les tentations qui lui font obstacle et mobiliser le courage nécessaire pour la poursuivre sans faille. Aux yeux de ses partisans, l'idée qu'il existe des principes de conduite valables indépendamment de notre volonté n'est d'ailleurs pas seulement fausse, mais pernicieuse. Qui refuse de faire usage de sa capacité d'autonomie est censé s'exposer à la tyrannie. Respecter quelque chose comme étant plus grand que nous revient, dit-on, à nous estimer nous-mêmes d'autant moins. Tout en constituant un idéal, la liberté humaine ainsi conçue n'est pourtant pas, il faut le souligner, en soi un idéal moral. Elle porte comme telle sur le rapport que chacun de nous a envers luimême, et non sur nos rapports envers autrui. Mais c'est précisément cette indépendance de ses attraits évidents à l'égard de la morale dont l'approche kantienne veut tirer parti. Elle vise à montrer que cet idéal de l'autonomie peut servir de fondement aux principes moraux qui ont pour objet de gouverner nos rapports avec d'autres individus.

Au fond, l'argument kantien se déroule ainsi : en exerçant notre autonomie, nous affirmons notre capacité de nous détacher des circonstances données pour nous gouverner d'après nos propres lumières. Ce que nous affïrmons par là n'est pourtant rien de particulier à nous-mêmes, car il s'agit d'une capacité possédée également par toute l'humanité. Ainsi, il s'ensuit, à ce qu'on suppose, que nous avons la même raison de respecter la liberté d'autrui que d'exercer la nôtre. L'autonomie kantienne, Alain Renaut par exemple l'a parfaitement démontré, est donc autre chose qu'un individualisme (et par conséquent la subjectivité moderne n'est pas faite d'une seule pièce) ${ }^{9}$ Et de ce premier principe d'une égale liberté pour tous découlerait ensuite tout un système de morale.

L'argument repose pourtant sur un sophisme. Comme il ressort tout de suite de l'ébauche précédente, mais on peut repérer l'erreur sans difficulté dans les versions plus détaillées des néo-kantiens eux-mêmes, l'argument passe sous silence le sens manifeste où ma capacité d'auto-détermination se distingue de celle d'autrui. Cet égard, bien entendu, c'est qu'elle est la mienne. Pourquoi ne faut-il pas tenir compte de ce fait?'(Car c'est assurément un fait.) Pourquoi serait-il exclu d'attacher une plus grande valeur à ma propre autonomie qu'à celle des autres? L'argument ne dispose pas luimême des moyens de répondre à ces questions ${ }^{10}$. Si je choisis d'accorder plus d'importance à ma propre liberté, je dois reconnaître, il est vrai, que les autres ont un droit pareil de faire de même. Mais de ce que j'admets que l'autre a une raison d'affirmer sa liberté, tout comme j'en ai une d'affirmer la mienne, il ne suit pas que je partage la raison qu'il a d'apprécier la sienne. Nos raisons sont pareilles, mais (chacune se spécifiant par rapport à l'agent qui l'a) elles ne reviennent pas, pour ainsi dire, à une raison unique.

Si je choisis en revanche de respecter la liberté des autres, lui accordant la même valeur qu'à la mienne, ce n'est pas l'intérêt que je porte à ma propre autonomie qui m'y pousse. Pour faire cette abstraction de soi, pour croire qu'il ne me faut pas me préférer aux autres simplement parce que je suis moi, je dois être déjà persuadé de la nécessité de les traiter d'égal à égal, et non

9. Voir Alain Renaut, L'ère de l'individu, Paris, Gallimard, 1989.

10. L'objection est bien connue (voir par ex. Bernard Williams, Ethics and the Limits of Philosophy, Cambridge, Mass., Harvard University Press, 1985, p. 60-63), mais il est nécessaire, semble-t-il, de la rappeler continuellement. 
comme des moyens ou des obstacles à l'exercice de ma propre liberté. Ce qui revient à dire que je dois avoir déjà adopté le point de vue moral et cela, indépendamment de mon souci d'autonomie.

Une fois de plus, il s'avère donc impossible de nous amener par la raison à la morale. Le motif fondamental d'être juste ne pèut se chercher dans le désir d'être autonome. Au contraire, ce n'est qu'en se situant à l'intérieur de la morale qu'on peut être incité à limiter sa liberté de façon qu'elle s'accorde avec une égale liberté d'autrui. Au lieu de prôner une morale de l'autonomie, et malgré les résonances qu'une telle éthique ne peut qu'éveiller dans une culture qui range la liberté parmi ses plus hautes valeurs, il faut conclure, à mon avis, à l'autonomie de la morale. De nos jours, aucun philosophe n'a exposé cette réorientation de la pensée morale avec autant d'éloquence et de persistance qu'Emmanuel Lévinas. « La priorité de l'éthique par rapport à l'ontologique "est la formule qu'il a choisie pour l'exprimer"

\section{IV}

Que la conscience morale dépose pour elle-même, sans être obligée de tirer son autorité de considérations extrinsèques, c'est là, on se le rappelle, la vérité dont Kant s'est avoué redevable à Rousseau. Ainsi, on pourrait se demander s'il a pu vraiment succomber à la sorte de tentation philosophique dont il se voulait guéri. A-t-il commis la double erreur de tomber dans le sophisme mentionné et cela, dans l'intention de faire ce qu'il n'aurait jamais dû tenter ? À vrai dire, Kant me paraît bien moins coupable à cet égard que d'autres, l'argument " kantien " ayant été plutôt développé par ceux qui se sont réclamés de lui. Depuis la préface à la Critique de la raison pratique du moins, il soutient sans la moindre ambiguité que l'autonomie ne peut servir de ratio cognoscendi de la loi morale ${ }^{12}$. Aussi n'a-t-il pas oublié, à ce qu'il paraît, la leçon du Vicaire savoyard.

Cela dit, il est pourtant évident que Kant accorde à la notion d'autonomie un rôle central dans son éthique. Elle constitue, dit-il dans cette même préface, la ratio essendi de la loi morale. Par là, Kant veut dire, il est clair, qu'on ne peut rendre compte de tout dévouement véritable à la morale qu'en y voyant un exercice d'auto-législation. Aussi impossible qu'il soit de justifier le point de vue moral par référence à l'autonomie, il n'existe à son avis d'autre façon de l'expliquer. Ressentir un attachement à la morale pour elle-même, seuls des êtres autonomes sont à même d'en faire preuve.

Sous ce jour, il n'est pas difficile de comprendre pourquoi d'autres penseurs croient s'inspirer de Kant lorsqu'ils veulent chercher dans l'autonomie le motif fondamental d'être juste. La raison que Kant donne en effet pour refuser de déduire la morale de l'autonomie n'est malheureusement pas le principe de Rousseau selon lequel le point de vue moral ne peut se justifier de l'extérieur. Il invoque, au contraire, l'impossibilité de savoir sur la base de l'expérience (seule source de la connaissance, insiste-t-il) que nous pouvons nous élever au-dessus de tout ce que

11. Emmanuel Lévinas, De Dieu qui vient à lidée, Paris, Vrin, 1992, p. 202.

12 Kant, Kritik der prakrischen Vernunft, Akademie-Ausgabe, Berlin, Preussische Akademie der Wissenschaften, 1900-1942, vol. 4, p. 4 et aussi p. 29. Pour l'évolution de ce point de vue chez Kant, voir Dieter Henrich, " Der Begriff der sittlichen Einsicht und Kants Lehre vom Faktum der Vernunft », dans Henrich et al. (éds.), Die Gegenwart der Griechen im neueren Denken. Festschrift für H.G. Gadamer, Tübingen, Mohr, 1960, p. 77-115. 
l'expérience a fait de nous, comme l'exige selon lui l'autonomie. Pour avoir le droit de nous croire capables d'autonomie, il faut le déduire de notre capacité à trouver un intérêt intrinsèque à remplir nos obligations morales. Or, en règle générale, les kantiens postérieurs, étant des esprits plus terre à terre, ne supposent pas que l'autonomie doive revêtir un caractère non empirique. Ainsi, ils prennent le chemin, menant de l'autonomie à la morale, que Kant lui-même croyait bloqué.

Comme je viens de le suggérer, le raisonnement que Kant opposait à cette démarche constitue un faux pas. L'ignorance où nous sommes de notre capacité d'autonomie n'aurait dû servir, chez lui, que d'argument supplémentaire. Sa réponse principale aurait dû être que la conscience ne peut chercher que dans elle-même l'autorité de la loi morale. Ainsi s'exprime celui qui a vraiment pris à coeur l'autonomie de la morale. Cependant, ma défense de cette position n'est pas encore achevée. Nous ne serons pas venus à bout de "la morale de l'autonomie " tant que nous n'aurons pas examiné l'idée que l'autonomie peut du moins servir à expliquer la nature de l'attitude morale, sinon à la justifier.

En principe, l'examen peut pourtant être bref. Si, comme on l'a montré. la volonté de se comporter en agent autonome ne suffit pas pour adopter le point de vue moral, l'autorité des principes définissant ce point de vue ne peut donc consister en ce qu'un tel agent se les impose à lui-même. Si l'autonomie, exercée avec esprit de suite, ne suffit pas pour justifier la morale, elle ne peut expliquer non plus ce qui constitue le propre du point de vue moral. Au contraire, les principes constitutifs de la morale doivent être tels qu'ils s’imposent à l'agent de l'extérieur, indépendamment de son statut d'être libre. Une fois qu'on tient compte de la véritable raison pour laquelle l'autonomie n'est pas à même de fournir la ratio cognoscendi de la morale, il est évident qu'elle est également incapable d'en former la ratio essendi.

J'imagine néanmoins que certains esprits ne seront pas, et à juste titre, entièrement satisfaits de cet argument, aussi valable qu'ils puissent l'estimer. Il s'agit en effet d'un argument négatif qui n'explique pas comment il faut comprendre l'idée selon laquelle les principes fondamentaux de la morale s'imposent à nous de l'extérieur. De surcroît, on peut s'inquiéter d'où, en raisonnant comme je le fais, je veux en venir. En plaidant pour l'autonomie de la morale par rapport à l'autonomie humaine, aurais-je l'intention de réintroduire une fondation théologique de la morale? (Ma référence approbatrice à Lévinas pourrait le suggérer.)

Il n'en est pourtant rien. Le soupçon, je vous l'assure, n'est pas fondé. Mais pour saisir pourquoi le rejet de l'autonomie humaine, comme fondement de la morale, ne nous ramène pas à l'hétéronomie divine, il convient de faire un pas en arrière. J'ai déjà évoqué la raison principale pour laquelle on a voulu faire de l'autonomie le motif fondamental de la morale : c'est le prestige immense dont jouit l'idéal de liberté dans notre culture. Mais il faut aussi mettre au jour les raisons qui depuis Kant ont poussé tant de gens à voir dans l'autonomie la seule explication possible de l'attitude morale. Sans la preuve que ces raisons ne sont pas réellement solides et qu'il existe une meilleure façon de comprendre ce qu'on a voulu ainsi expliquer, l'autonomie de la morale - la thèse que je défends - ne paraîtra sans doute pas très convaincante. 
Évidemment, on a fait appel à une multitude d'arguments différents pour soutenir que le point de vue moral consiste dans l'activité de la raison à s'imposer à elle-même des règles de conduite. Mais à mon avis le seul argument sérieux est celui qui prétend ainsi sauvegarder l'objectivité de la morale. Au fond, cet argument provient de la volonté d'éviter deux autres points de vue ${ }^{13}$. D'un côté, il s'agit de rejeter ce qui s'appelle en général une conception expressiviste de la morale, selon laquelle tout jugement moral ne fait qu'exprimer des préférences de celui qui l'émet. Conception qui peut prendre des formes raffinées, les préférences en jeu étant supposées non seulement impersonnelles mais éventuellement aussi d' ordres " différents. Ainsi, l'analyse expressiviste veut rendre compte du fait que d'ordinaire on croit qu'une norme morale serait valable même si on ne l'acceptait pas : cette croyance ne serait que l'expression du désir (de deuxième ordre) qu'on a de se soumettre à cette norme même en l'absence d'un désir (de premier ordre) de le faire $e^{14}$. Pourtant, cette explication de l'objectivité visée par les jugements moraux ne satisfait pas nos néo-kantiens. L'idée que la distinction entre le juste et l'injuste est indépendante de nos préférences dans la mesure simplement où nous préférons qu'elle le soit - car c'est de cela qu'il s'agit ne paraît pas accorder à la morale la sorte d'objectivité qu'elle mérite. On aura réduit la morale à une affaire de préférences, nonobstant la hiérarchie qu'on en postule. À celui qui refuse d'accepter une certaine norme, on ne pourrait dire qu'il a tout de même raison de l'adopter, mais seulement qu'on préfêre qu'il l'adopte. À mon avis, les néo-kantiens ne se trompent pas du tout en trouvant cette conception intenable.

Mais s'ils excluent l'expressivisme d'un côté, ils repoussent, de l'autre, toute conception de l'objectivité morale qui assimile les jugements moraux au modèle habituel de la connaissance. Un jugement moral est raisonnable, insistent-ils, non en tant qu'il vise un domaine indépendant de faits (en l'occurrence de faits moraux), mais en tant qu'il est le résultat d'une procédure de construction, dans laquelle est censée consister évidemment notre autonomie. Or, pourquoi cette opposition à ce qui peut s'appeler une conception réaliste de la morale ? Deux considérations semblent décisives. Premièrement, ne serait-il pas bizarre, voire philosophiquement irresponsable, de supposer que le monde englobe, en plus des faits physiques et psychologiques que chacun tient pour des objets de la connaissance, une troisième dimension de faits moraux? Et puis, ne se ferait-on pas une image trop pauvre, trop passive, de l'agent moral, en le réduisant ainsi au profil du sujet de la connaissance, alors qu'en réalité il est justement un sujet de l'action? Si les jugements moraux ont pour fonction de régler notre conduite, ne se comprennent-ils pas mieux en termes faisant appel à la façon dont nous nous réglons nous-mêmes?

Ces deux arguments ne sont pourtant pas aussi forts qu'on les suppose, et à mon avis c'est du côté du réalisme moral que la vérité se trouve. L'idée qu'il existe des faits moraux n'aura rien de singulier, tant qu'on n'y voit plus (comme le plus souvent ses détracteurs) la notion qu'on pourrait observer des

13. Le livre récent de Christine Korsgaard, The Sources of Normativity, fournit un bon exemple de cette stratégie d'argumentation.

14. Cette analyse se trouve, par exemple, chez Alan Gibbard, Wise Choices, Apt Feelings, Cambridge, Mass., Harvard University Press, 1990, p. 164-170. 
qualités morales des choses, tout comme on peut observer leurs qualités physiques ou psychologiques. Ce n'est ni par la perception ordinaire ni par une faculté mystérieuse d'c intuition "qu'on découvre des faits moraux, mais par la réflexion, parce que ces faits consistent en l'existence d'une certaine sorte de raisons d'action. Les faits moraux qui composent sa situation, on les saisit en réfléchissant aux raisons qu'on a d'agir là d'une façon ou d'une autre. Si le réalisme moral se conçoit ainsi, il ne forme en rien une position bizarre ou irresponsable. Il implique d'ailleurs (et c'est un de ses mérites, j'en suis persuadé) que les faits moraux sont une espèce parmi d'autres d'une classe plus générale, qui est celle des faits normatifs. Car en plus des raisons d'agir (dont certaines constituent des faits moraux), il existe aussi des raisons de croire, des raisons auxquelles on fait appel pour justifier l'adoption de telle ou telle croyance. Elles aussi, on les découvre par le moyen si peu ésotérique qu'est la réflexion.

Les philosophes, il est vrai, n'ont d'ordinaire pas grand-chose à dire au sujet de la réflexion comme organe de la connaissance. Mais c'est là un véritable scandale, puisqu'il n'est pas d'autre moyen pour la philosophie ellemême d'arriver à constituer une forme de connaissance. Elle est, après tout, une discipline nécessairement normative, où il s'agit de la façon dont il faudrait comprendre les formes fondamentales de pensée dont nous faisons ou devrions faire usage.

Encore n'est-ce pas, je crois, par simple négligence qu'on hésite à aborder la question de savoir comment la réflexion peut fonctionner comme mode de connaissance. C'est que la nature d'une raison, qui est l'objet de la réflexion, s'avère un problème épineux. À strictement parler, des raisons de croire ne sont pas, par exemple, les autres croyances, en tant que telles, qu'on a et qui font partie de la justification d'une certaine proposition. C'est le rapport que ces croyances ont à la proposition en jeu, la mesure où elles sont pertinentes à la question de savoir si cette proposition est vraie ou fausse, qui font d'elles des raisons. Tout comme la souffrance d'autrui, qui en tant que telle consiste en un état psychologique de l'autre, n'est pas ellemême la raison d'intervenir, mais en constitue une par le rapport qu'elle a à nos possibilités d'action. En même temps, ce caractère " relatif "de toute raison d'agir ou de croire ne signifie pas que les raisons soient subjectives. On peut très bien se tromper sur les raisons qu'on a de faire ceci ou de croire cela, les rapports entre certaines données et des actions à faire ou des propositions à adopter - rapports en lesquels consistent les faits normatifs - existant indépendamment de nos opinions à leur égard.

Somme toute, une raison n'est donc pas une entité physique ou psychologique, mais une entité d'un troisième ordre, “ idéel " si l'on veut. Cette sorte de platonisme pose, sans doute, un problème pour tous ceux qui souscrivent à la conception du monde tellement répandue de nos jours - à savoir le naturalisme, selon lequel il n'existe que des faits physiques et psychologiques, qui sont l'objet des sciences naturelles. Mais à mon avis, il s'agit là d'une véritable idéologie, tant qu'on la partage sans vraiment prendre au sérieux les difficultés manifestes auxquelles elle est exposée. S'il n'existe en effet pas de faits authentiquement normatifs, comment peut-on dire des croyances normatives, c'est-à-dire des croyances affirmant qu'il faut croire ceci ou faire cela, qu'elles sont vraies ou fausses? Comment peut-on done même parler en ce cas de croyances? Et si l'on voit surtout dans les jugements normatifs concernant ce qu'il faut croire, non l'expression de croyances elles-mêmes capables d'être vraies ou fausses, mais l'expression de 
préférences, de la même façon que l'expressivisme comprend les jugements pratiques ou moraux (et comment le naturaliste peut-il en toute rigueur procéder autrement), que fait-on sinon sombrer dans l'irrationalisme le plus pur et dur?

Ces objections, je les ai développées ailleurs ${ }^{15}$. À présent, il faut retourner à nos kantiens. Or, il me semble que, malgré leur opposition à l'analyse expressiviste de la morale, ils restent eux-mêmes trop prisonniers de cette conception naturaliste du monde. Eux aussi, ils regardent comme allant de soi qu'il ne peut exister de faits normatifs indépendants que notre raison aurait pour tâche de découvrir. Ainsi, voulant tout de même maintenir une sorte d'objectivité pour la morale, ils s'efforcent de voir dans la normativité quelque chose que la raison construit. J'ai déjà expliqué pourquoi cette stratégie constructiviste ne peut réussir. Mais il sera peut-être utile d'illustrer l'erreur par un exemple.

Examinons donc la thèse constructiviste dans la forme que Christine Korsgaard lui donne. Tout impératif pratique, écrit.elle, « ne repose pas sur la reconnaissance d'un fait normatif ou vérité normative, mais seulement sur la nature de la volonté [...] L'argument kantien part donc de la nature de la volonté rationnelle pour arriver à un principe, qui décrit une procédure, selon laquelle une telle volonté doit procéder et de là à une application du principe "dans un impératif pratique ${ }^{\mathrm{k}}$ Comme le précise la deuxième phrase de la citation, les impératifs pratiques sont censés provenir, non de la volonté comme telle (à l'encontre de ce que la première phrase suggère), mais de la volonté rationnelle. C'est là pourtant que gît la difficulté. Une volonté n'est rationnelle que dans la mesure où elle possède des propriétés qu'une volonté devrait posséder. Il s'ensuit que les vérités normatives fixant ces propriétés sont préalables à toute procédure pour construire des normes. Le constructivisme, comme théorie générale de la normativité, n'est donc pas tenable. Évidemment, certaines normes peuvent tirer leur origine de la volonté rationnelle, leur autorité consistant en la décision de se les imposer. Mais il n'en peut être ainsi dans le cas de toutes, ni des plus fondamentales. Selon Korsgaard, il faut adhérer au " réalisme de procédure "de type kantien, selon lequel " il existe des réponses à des questions morales, parce qu'il existe des procédures correctes pour les déterminer». Mais la vérité se trouve, en dernière instance, du côté du "réalisme de substance "qu'elle rejette, selon lequel " il existe des procédures correctes pour répondre aux questions morales parce qu'il y a des vérités morales ou faits moraux qui existent indépendamment de ces procédures". Comment peut-on autrement comprendre l'idée de " procédure correcte»? Ce n'est pas Kant, mais Platon qui s'avère - à cet égard du moins - le guide le plus sûr.

Qu'en est-il pourtant de l'autre considération qui semble, aux yeux des kantiens, s'opposer à toute conception réaliste de l'objectivité morale ? À savoir le reproche d'avoir réduit les convictions morales à une espèce de connaissance, alors qu'elles ont plutôt la nature d'engagements pratiques? Or, cette critique n'est pas plus valable que le contraste entre théorie et pratique sur lequel elle repose. Elle suppose notamment, et c'est là une supposition partagée par de nombreux philosophes depuis Hume, que

15. Voir Larmore, The Morals of Modernity, Cambridge, Cambridge University Press, 1996, chapitre V, et aussi Modernité et morale, Paris, Presses Universitaires de France, 1993, chapitre $\mathrm{I}$, où j'analyse en détail les différentes formes du naturalisme dans l'éthique contemporaine.

16. Korsgaard, The Sources of Normativity; p. 36. 
du point de vue de la motivation les croyances sont inertes et qu'elles ne peuvent pas nous pousser à l'action sans l'apport du facteur supplémentaire qu'est la volonté ou le désir. Mais les croyances normatives constituent précisément un contre-exemple à cette idée préconçue ${ }^{17}$ Ce sont là des croyances qui en général prétendent en même temps être vraies - qu'elles portent sur la façon dont il faut former des croyances ou choisir des actions - et régler à cet égard notre conduite. Ainsi, les jugements moraux, en particulier, peuvent exprimer des comnaissances tout en nous engageant aussi dans la pratique.

Un réalisme (ou platonisme, si l'on veut) qui rapporte la morale à un domaine de faits moraux indépendants de notre volonté ne donne donc pas une image trop passive de l'agent moral. Ce sont plutôt les kantiens qui, comme tant d'autres, s'appuient sur une conception trop passive de ce que peut ètre la connaissance. En disant cela, il faut le signaler, je n'invoque aucune notion hétérodoxe de la connaissance. Je fais appel à une vérité que chacun sera prêt à admettre, s'il n'est pas entravé par certains préjugés philosophiques - à savoir que nous pouvons nous comporter d'une certaine façon, dans nos pensées comme dans nos actions, tout simplement parce que nous croyons qu'il le faut.

Ces remarques auront donc suffi, je l'espère, pour refroidir tout enthousiasme spontané qu'on pouvait toujours éprouver pour la morale de l'autonomie. Et aussi pour assurer les esprits inquiets que le rejet de cette conception ne signifie pas un retour à l'hétéronomie divine. En guise de conclusion, je veux situer plus exactement dans l'espace philosophique la thèse de l'autonomie de la morale que j'ai défendue tout au long de cet essai. À cette fin, il faut retourner à l'idéologie naturaliste dont il était question plus haut. Le naturalisme, dans le contexte de la philosophie morale, signifie deux doctrines un peu différentes, mais qui sont souvent et facilement embrassées à la fois. Il est utile de les distinguer, même s'il s'agit pour moi de les rejeter toutes les deux.

En premier lieu, le naturalisme représente une conception du monde, en ce sens qu'il délimite les sortes de choses qui s'y trouvent et peuvent devenir des objets de la connaissance. C'est là le sens du terme que nous avons déjà rencontré. Selon le naturalisme, le monde ne serait que matière et mouvement, avec les pensées et sentiments que nous avons à son égard. Les analyses expressivistes de la morale, il est clair, reposent sur cette conception du monde. Mais s'y fonde aussi la morale de l'autonomie d'inspiration kantienne, tant qu'elle écarte comme extravagante toute idée de faits moraux existant indépendamment de la volonté rationnelle.

Le naturalisme en ce sens est intenable, je crois l'avoir démontré, pour la raison fondamentale que le monde ainsi conçu n'a pas de place pour des entités telles que des raisons qui, n'étant ni physiques ni psychologiques en caractère, constituent un troisième ordre de faits, irréductiblement normatifs. Il faut préciser : je ne dis pas que l'abandon de cette sorte de naturalisme

17. Cette thèse aussi, je l'ai exposée plus amplement ailleurs. Voir les références données dans la note 16 et aussi mon essai - Denken und Handeln - dans Deutsche Zeitschrift für Philosophie, vol. $45(1997) n^{\circ} 2$, p. 183-195. 
suffise pour justifier l'autonomie de la morale. Tant s'en faut, il est possible d'admettre l'existence objective d'entités telles que des raisons tout en voulant expliquer l'autorité de la morale par des considérations indépendantes, en l'occurrence par des raisons d'action préalables au point de vue moral. Ce que je soutiens, c'est que, une fois reconnue la validité de cette idée non naturaliste du monde, on aura surmonté un des principaux obstacles à la reconnaissance de la véritable nature de la morale.

Dans un deuxième sens, le naturalisme s'oppose plus directement à l'autonomie de la morale. Il s'agit alors, non d'une image du monde, mais pour ainsi dire d'une conception de l'agent en face du monde, conception psychologique qui veut minimiser, sinon nier, l'apport distinctif du point de vue moral. Selon l'expression de Bernard Williams (qui paraît bien disposé à son égard), " une psychologie morale naturaliste explique nos capacités morales en termes de structures psychologiques qui ne sont pas en soi morales ". Williams continue : " $\grave{\Lambda}$ la question "combien nos explications de l'activité morale devraient-elles ajouter à nos explications d'autres activités humaines'?", elle répond " aussi peu que possible" " $"$.

À l'évidence, c'est précisément cette conception de la psychologie humaine que je tiens à rejeter. Je ne dispose d'aucun argument général pour démontrer au contraire l'autonomie de la morale. L'existence d'un tel argument paraît même exclue par la thèse que la morale parle pour ellemême. En fin de compte, la seule façon générale de reconnaître cette vérité consiste à écouter sa propre conscience - ce qui est loin, bien entendu, de constituer un argument. Mais en revanche il est possible de montrer comment des tentatives particulières pour ramener l'autorité de la morale à des considérations extrinsèques ne réussissent pas. C'est ce que j'ai fait dans le cas des approches hobbésienne et kantienne. On peut aussi demander quel intérêt porte tant de gens à croire que la morale ne constitue pas un ordre autonome, mais doit se légitimer à partir d'un point de vue extérieur. S'agit-il d'un goût pour la simplicité conceptuelle? Ou faut-il chercher les racines de cette attitude à un niveau plus profond?

De toute façon, la question primordiale qui donne naissance à toute cette problématique - à savoir " Pourquoi faut-il être juste? — - n'a rien pour encourager le naturalisme en ce sens. Bien au contraire, je l'ai fait remarquer au début, cette question surgit dans un contexte où l'on partage déjà le point de vue moral, mais où l'on se sent tiraillé aussi par d'autres intérêts. Rien dans une telle situation n'oblige qu'on cherche des raisons pour décider s'il faut adopter le point de vue moral en tant que tel. Le problème consiste plutôt à mettre en balance deux engagements auxquels on est attaché.

Je veux souligner que la réponse qu'il faut donner à ce problème-là, dans chaque contexte où il se pose, n'est pas évidente. Je ne crois même pas qu'on puisse y répondre d'une façon globale. La raison en est que même si la morale constitue une forme de valeur irréductible - c'est la thèse que je défends -, elle n'est pas pour autant une valeur toujours suprême. Il n'est pas exclu que, tout en admettant l'autorité intrinsèque de la conscience morale, nous concluions que, du moins dans la situation donnée, elle exige trop de nous. S'il faut refuser de réduire la morale à nos autres intérêts, il

18. Bernard Williams, * Nietzsche's Minimalist Moral Psychology ", p. 67.68 dans son Making' Sense of Humanity, Cambridge, Cambridge University Press, 1995. 
faut aussi se retenir de croire qu'elle doive toujours l'emporter sur ces intérêts. Nous devons reconnaître une vérité que notre tradition philosophique a toujours eu peine à accepter : la réflexion pratique, même en dernière instance, ne parle pas d'une seule voix, morale ou autre.

Département de philosophie

Université de Chicago 